\title{
Fuzzy Classifier for Mental Stress Estimation using ECG Statistical Parameters
}

\author{
Sneha Mittal ${ }^{1}$ and Nirmal Singh Grewal ${ }^{2}$ \\ 1 (ECE Deptt., GNE College, Ludhiana, Punjab INDIA, \\ ${ }^{2}$ (Deptt., GNE College, Ludhiana, Punjab INDIA,
}

\begin{abstract}
Mental Stress estimation is an important feature to be derived in health related diagnostic activity. It has been observed that the stress has a major effect on heart functioning. And therefore, ecg should be the major source of stress variation and can be analyzed in various ways in order to extract the effect of mental stress. In the presented work, the ecg is analyzed using the statistical parameters set (energy, entropy, power, standard deviation and covariance). The parameters are not directly computed form the ecg itself. The ecg is first decomposed to level-2 using BIOR-3.9 wavelet transform to reduce the dimensionality of the ecg sample size. The level-1 and level-2 parameters are used to derive the mental stress levels as normal $(\mathrm{N})$, hyper-1 (H-1), hyper-2 (H-2), depression-1 (D-1) and depression-2 (D-2). On parameter analysis, it has been observed that the energy and entropy are the two parameters that show an effective variation in values when normal to depression or normal to hyper case is observed. Therefore, the energy and entropy values are used for rule making and learning of the system in order to derive the mental stress levels
\end{abstract}

\section{Keywords}

ECG, BIOR-3.9 wavelet, Entropy, Energy, Power, Standard Deviation, Covariance, Fuzzy Logic, Mental Stress

\section{INTRODUCTION}

ECG is the most sensitive human body parameter that is affected most due to mental stress. However, in case of normal or hyper cases, the stress can be seen on faces and does not require the ecg analysis. The presented work finds application in analyzing the patients mental condition wherever he may respond or not respond to stress if there. e.g. patients in deep coma or patients suffering from severe paralytic attack. The ecg is the best part to assess the stress as it is most affected from normal to stress like conditions.

The presented work is organized in four sections: 1) ECG Decomposition, 2) Parameters computation, 3) Fuzzification of parameters 4) Stress Estimation. The algorithm discussed in subsequent section is implemented using matlab 7.5 version. Sanp shots and results are given in subsequent sections.

\section{RELATED WORKS}

Fetal ECG analysis is a very sensitive issue in analyzing the featus cardiac activity inside the mothers tomb. Accurate identification of featus ecg from mother's ecg is an interesting and challenging target. And to extract inference from it is more tedius task. This requires a lot of study and data base to validate the results [1].

Wearable ECG sensors are good option for prolonged monitoring of cardiac patients. Compression of ecg signal using wavelet analysis is discussed heer. [2].
A wavelet-based method for the compression of electrocardiogram (ECG) signal is also prepared. The discrete wavelet transform (DWT) is applied to the digitized ECG signal; then the resulting wavelet coefficients are threshold using a threshold based on energy packing efficiency of the wavelet coefficients. To assess the technique properly we have evaluated the effect of threshold levels selection on the quality of the reconstructed signal. The performance parameters of the compression algorithm are measured and a compression ratio of 14.55:1 with percent root mean square difference (PRD) of $2.56 \%$ is achieved [3].

Emotional stress refers to the inducement of stress due to the consequence of a continuous experience of negative emotions (sad, anger, fear and disgust). This work aims to investigate the effect of negative emotions in emotional stress inducement through Electrocardiogram (ECG). 20 university students with a mean age of 24-years have participated in this study. Perceived Stress Scale (PSS-10) questionnaire is used to evaluate the subject's initial stress behavior.

Self Assessment Manikin (SAM) and Self Assessment Form is used to evaluate the subject emotional response during and after the data acquisition, respectively. Statistical features such as heart rate (HR), approximate entropy (Ap En), mean R amplitude (MRA), mean R-R interval (MRRI), standard deviation of normal to normal RR intervals (SDNN) and root mean square of successive heartbeat interval differences (RMSSD) are extracted from HRV signals. Finally, the emotional stress assessment on this work consists of two stages namely; three valence classification (positive emotions, negative emotions and neutral) and emotional stress classification where negative emotions are further classified into either emotional stress or non emotional stress. To visually observe the distance between each class, Fuzzy C Means (FCM) Clustering plot is implemented and Euclidean distance measure.[4]

QRS complex is the most important wave in the entire ecg, Nomally $\mathrm{R}$ is the peak in the ecg and depicts the heart rate of the person under scanner. QRS width also gives a vital information about the pumping capacity of the heart. The entire P-QRS-T-U wave complex may be brought under geometrical analysis for predicting and analyzing the heart condition.[5]

A novel method of HRV analysis for mental stress assessment using fuzzy clustering and robust identification techniques is discussed here. The approach consists online monitoring of heart rate signals, signal processing, exploiting fuzzy clustering and fuzzy identification techniques to render robustness and monitoring the functioning of autonomic nervous system under different stress conditions. [6].

Fourier transform is the most useful tool in analyzing the ecg in frequency domain. As the Fourier analysis decomposes the given signal into its frequency components, the ecg is also composed of different $r=$ frequency component and the frequency domain analysis reveals vital information about the heart pumping.[7] 
After the Fourier transform analysis of ecg, the wavelet based analysis is very common. There are different wavelet families where the ecg can be decomposed into approximates and details component and that at reduced dimension. This makes the analysis fast and accurate. [8].

ECG shows the electrical actibvity of the heart operation. A web application extracts the features of ECG signal like ST segment, QRS wave, etc. and use these features for identifying whether a person suffers from any of the four levels of stress, that is, Hyper Acute stress (Myocardial Infarction), Acute stress (Type A), Hyper Chronic stress (Ischemia) or Chronic Stress (Type B) [9].

\section{Methodology}

ECG is the electrical activity of the heart functioning measured in terms of millivolt signals generated at different nodes in the human body. When plotted the millivolt signal against time on a graphical sheet, it is termed as ecg signal plot. An ecg consists of $\mathrm{P}-\mathrm{QRS}-\mathrm{T}-\mathrm{U}$ waves and one complete set of P-QRS-T-U wave is called as one ecg complex.

Normally R-wave has the highest peak in the ecg complex and the frequency of R-wave gives the heart rate of the person under scanner.

The arrival of P-QRS-T-U wave with time is an important parameter while analyzing the health condition of the person under scanner. An ecg complex is shown in below given image.

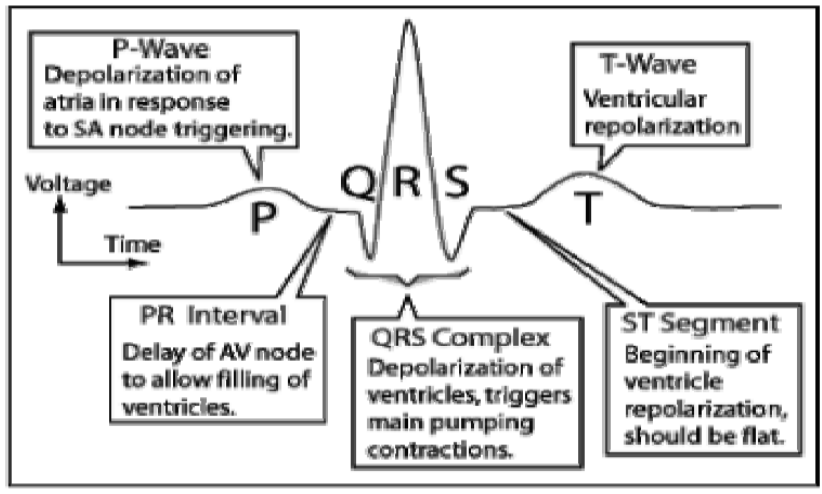

\section{Algorithm}

MIT data base of ecg has been used for testing of the presented algorithm. A real time ecg is also extracted using the single lead ecg machine for testing purposes. The ecg under test is brought under decomposition using BIOR-3.9 wavelet family upto 3levels. The original ecg signal and after decomposed using bior3.9 wavelet family at level-1 is shown below:

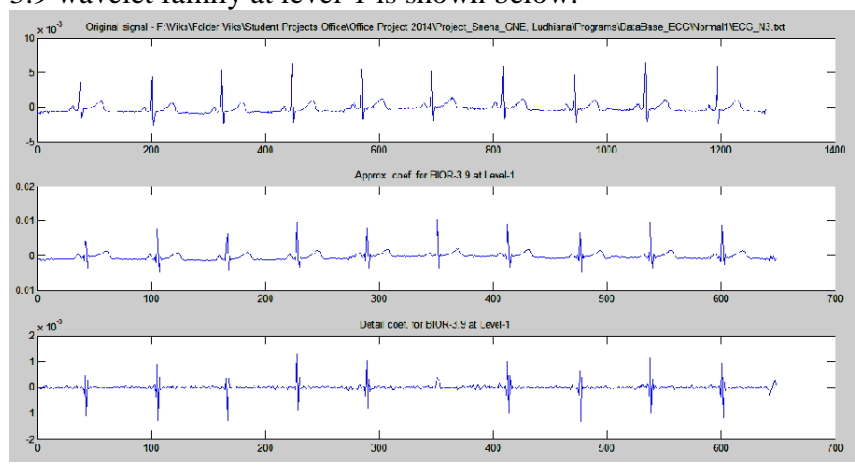

Following parameters are computed after decomposing the ecg signal upto level-3:

\section{Entropy (Ent)}

The entropy of a signal is expressed as the measure of the randomness. Entropy seems to be effective in dealing with complex biological signals. The entropy (Ent) is given by:

$$
\text { Entropy }(E n t)=-\sum_{i=1}^{n} p(x i) \cdot \log _{10} p(x i)
$$

Where, $\mathrm{x}=\left(\mathrm{x}_{1}, \mathrm{x}_{2} \ldots \mathrm{x}_{\mathrm{n}}\right)$ is a set of wavelet co-efficient, and $\mathrm{p}$ is a probability of wavelet co-efficient.

\section{Power (P)}

Power is defined as an average square sum of wavelet coefficients and given by the following equation:

$$
\operatorname{Power}(P)=\frac{1}{n} \sum_{i=1}^{n} x_{i}^{2}
$$

Where xi and $\mathrm{n}$ are the wavelet coefficient and total no of wavelet coefficients respectively.

\section{Energy (E)}

Energy is expressed as square sum of wavelet coefficients and given by the following equation:

$$
\operatorname{Energy}(E)=\sum_{i=1}^{n} x_{i}^{2}
$$

Where xi and $\mathrm{n}$ are the wavelet coefficient and total no of wavelet coefficients respectively.

\section{Mean $(\mu)$}

Mean is computed by average sum of wavelet coefficients and given by the following equation:

$$
\operatorname{Mean}(\mu)=\frac{1}{n} \sum_{i=1}^{n} x_{i}
$$

Where $x i$ and $n$ are the wavelet coefficient and total no of wavelet coefficients respectively.

\section{Standard Deviation (SD)}

Standard Deviation is expressed as:

$$
S D=\frac{1}{n} \sum_{i=1}^{n}\left(x_{i}-\mu\right)^{2}
$$

Where $\mathrm{xi}$ and $\mathrm{n}$ are the wavelet coefficient and total no of wavelet coefficients respectively.

\section{Fuzzy Classifier}

The parameters suggested in the previous sections are computed using matlab 7.5 version. The results are given in the table given in result section. It is observed from the result table that at each decomposition level, the energy and entropy are the most sensitive parameters that shows a very clear distinction between a stressed state and normal state of mind. Therefore, the entropy and energy may further be used to resolve different stress states within stressed and normal.

Further, the energy and entropy values at each three levels are normalized between -1 to +1 range crossing zero. This establishes the fuzzy association or membership of energy and entropy values to classify the mental stress.

The maximum and minimum values are taken as +1 and -1 and the mid values are computed proportionately. 


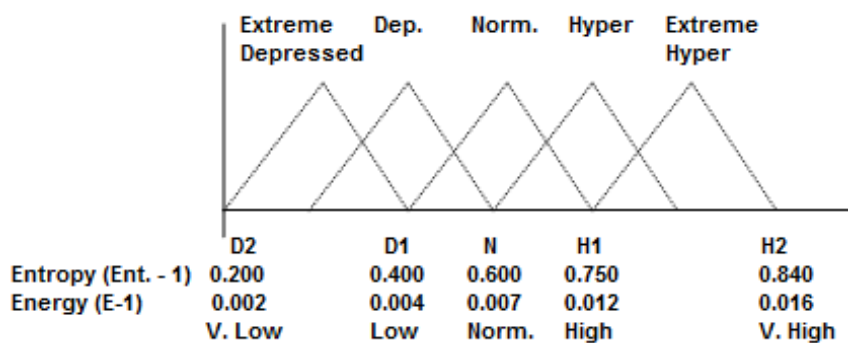

Fuzzy Association of Energy and Entropy with Mental Stress at Level-1 Decomposition

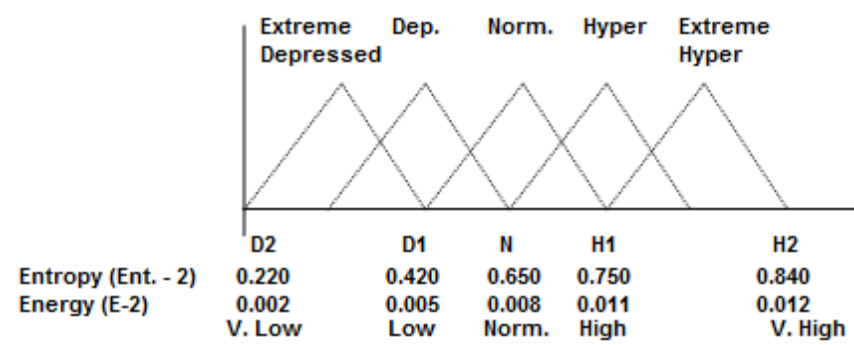

Fuzzy Association of Energy and Entropy with Mental Stress at Level-2 Decomposition

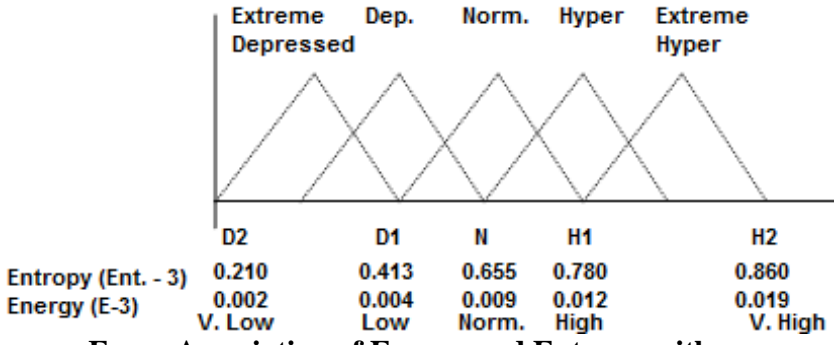

Fuzzy Association of Energy and Entropy with

Mental Stress at Level-3 Decomposition

In the presented work, stress states are divided as under given below:

$\begin{array}{ll}\text { Hyper } & \rightarrow \mathrm{H}-2 \\ \text { Hyper } & \rightarrow \mathrm{H}-1 \\ \text { Normal } & \rightarrow \mathrm{N} \\ \text { Depressed } & \rightarrow \mathrm{D}-1 \\ \text { Depressed } & \rightarrow \mathrm{D}-2\end{array}$

The fuzzy membership association of energy and entropy at different levels is shown in the figure.

\section{RESUlts}

The presented algorithm has been tested on ecg of different persons and the data base used is from MIT data base. The MIT data base includes normal and stressed person. The stressed person data base is based on person driving the truck on busy road. Following tables shows the values of entropy and energy at different levels. The range form the table are used for making rule-base for different states of mental stress levels as shown in figure.

Normal ECG

\begin{tabular}{|c|c|c|c|c|}
\hline S.No. & \multicolumn{2}{|c|}{ Level-1 } & Level-2 & Level-3 \\
\hline \multirow{2}{*}{1} & Energy & 0.007 & 0.0076 & 0.008 \\
\cline { 2 - 5 } & Entropy & 0.600 & 0.623 & 0.633 \\
\hline 2 & Energy & 0.0067 & 0.008 & 0.0073 \\
\hline
\end{tabular}

\begin{tabular}{|c|c|c|c|c|}
\hline & Entropy & 0.624 & 0.633 & 0.678 \\
\hline \multirow{3}{*}{3} & Energy & 0.007 & 0.0076 & 0.0078 \\
\cline { 2 - 5 } & Entropy & 0.631 & 0.663 & 0.671 \\
\hline
\end{tabular}

\begin{tabular}{|c|c|c|c|c|}
\multicolumn{5}{c}{ Stressed ECG } \\
\hline S.No. & \multicolumn{2}{|c|}{ Level-1 } & Level-2 & Level-3 \\
\hline \multirow{2}{*}{1} & Energy & 0.002 & 0.0023 & 0.003 \\
\cline { 2 - 5 } & Entropy & 0.200 & 0.210 & 0.213 \\
\hline \multirow{2}{*}{2} & Energy & 0.0015 & 0.0013 & 0.0031 \\
\cline { 2 - 5 } & Entropy & 0.202 & 0.230 & 0.219 \\
\hline \multirow{2}{*}{3} & Energy & 0.0015 & 0.0022 & 0.0029 \\
\cline { 2 - 5 } & Entropy & 0.203 & 0.212 & 0.203 \\
\hline
\end{tabular}

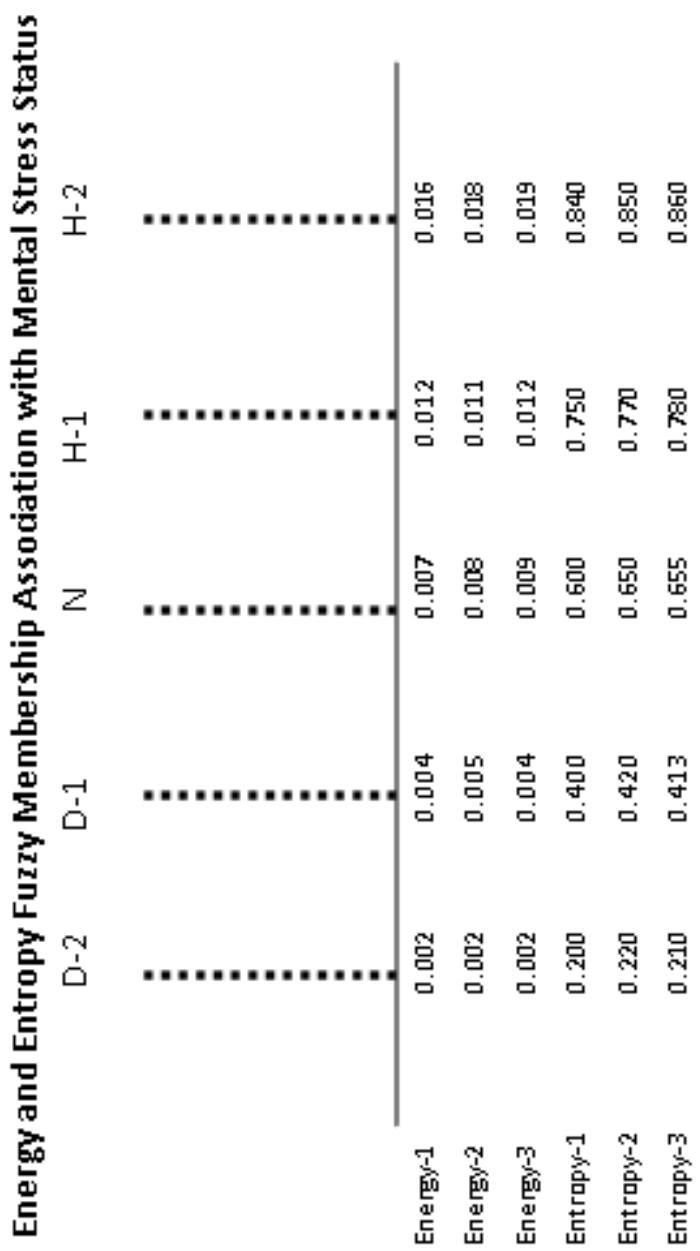

\section{Conclusion}

The result table indicates that the ecg signal can be very well resolved to define different mental stress states of a person under scanner. The presented algorithm needs to be tested on large no. of data base of ecg for validation purposes. Then only a strong correlation could be established between the energy values and entropy values to that of mental stress.

As listed above more ecg parameters, only energy and entropy are the parameters that are affected most when a person's stress state varies. The sensitivity of other parameters like mean, 
standard deviation etc. can only be adjudged when the algorithm is tested on large no. of ecg data base and that too on real time ecg signals.

\section{REFERENCES}

[1] Zhilin Zhang_, Student Member, IEEE, Tzyy-Ping Jung, "Compressed Sensing for Energy-Efficient Wireless Telemonitoring of Non-Invasive Fetal ECG via Block Sparse Bayesian Learning", ACCEPTED BY IEEE TRANSACTIONS ON BIOMEDICAL ENGINEERING, 2012

[2] Fahimeh Ansari-Ram, Saied Hosseini-Khayat, "ECG Signal Compression Using Compressed Sensing with Nonuniform Binary Matrices”, 978-1-4673-1479-4/12/\$31.00 (02012 IEEE

[3] M. Sharafat Hossain , "ECG Signal Compression using Energy Compaction Based Thresholding of the Wavelet Coefficients”, DUET Journal Vol. 1, Issue 2, June 2011.

[4] Bong Siao Zheng, M Murugappan and Sazali Yaacob, "FCM Clustering of Emotional Stress using ECG Features", International conference on Communication and Signal Processing, April 3-5, 2013, India.

[5] Mohammad Reza Homaeinezhad1, 2, Ehsan Tavakkoli1,2 , Ali Ghaffari, "Discrete Wavelet-based Fuzzy Network Architecture for ECG Rhythm-Type Recognition: Feature
Extraction and Clustering- Oriented Tuning of Fuzzy Inference System" , International Journal of Signal Processing, Image Processing and Pattern Recognition Vol. 4, No. 3, September, 2011.

[6] Mohit Kumar, Matthias Weippert, Reinhard Vilbrandt, Steffi Kreuzfeld, and Regina Stoll, "Fuzzy Evaluation of Heart Rate Signals for Mental Stress Assessment" , IEEE TRANSACTIONS ON FUZZY SYSTEMS, VOL. 15, NO. 5, OCTOBER 2007.

[7] G.Ranganathan, V.Bindhu, Dr.R.Rangarajan, s "ECG Signal Processing using Dyadic wavelet for Mental Stress Assessment", 978-1-4244-4713-8/10/\$25.00 @2010 IEEE.

[8] C. Saritha, V. Sukanya, Y. Narasimha Murthy, "ECG Signal Analysis Using Wavelet Transforms", Bulg. J. Phys. 35 (2008) 68-77. Prof, Shamla Mantri, Dr. Pankaj Agrawal, Prof. Dipti Patil, Dr. V. M. Wadhai

[9] "Depression Analysis using ECG Signal”, ISSN 2277-3061, Nov 10,2013

Author's Profile Sneha Mittal ${ }^{1}$ has received the B.Tech Degree in EIE from,IITT POJEWAL, Punjab in 2005 and pursuing his M.Tech in ECE from GNDEC Ludhiana, Punjab.. Her field of interest is in Bio medical based application system developments. 